\title{
Surgical and Oncological Outcomes of Robotic Resection for Sigmoid and Rectal Cancer: Analysis of 109 Patients from A Single Center in China
}

Jianhong Peng

Sun Yat-sen University Cancer Center

Weihao Li ( $\nabla$ liwh1@sysucc.org.cn)

Sun Yat-sen University Cancer Center https://orcid.org/0000-0003-4593-4264

Jinghua Tang

Sun Yat-sen University Cancer Center

Yuan Li

Sun Yat-sen University Cancer Center

\section{Xueying Li}

Sun Yat-sen University Cancer Center

Xiaojun Wu

Sun Yat-sen University Cancer Center

Zhenhai Lu

Sun Yat-sen University Cancer Center Junzhong Lin

Sun Yat-sen University Cancer Center

Zhizhong Pan

Sun Yat-sen University Cancer Center

\section{Research}

Keywords: sigmoid cancer, rectal cancer, robotic surgery, surgery outcome, oncological outcome, neoadjuvant chemoradiotherapy

Posted Date: January 22nd, 2021

DOl: https://doi.org/10.21203/rs.3.rs-150874/v1

License: @ (1) This work is licensed under a Creative Commons Attribution 4.0 International License. Read Full License 


\section{Abstract}

Background: Robotic colorectal surgery has been increasingly performed in recent years. The safety and feasibility of its application has also been demonstrated worldwide. However, limited studies have presented clinical data for patients with colorectal cancer (CRC) receiving robotic surgery in China. The aim of this study is to present short-term clinical outcomes of robotic surgery and further confirm its safety and feasibility in Chinese CRC patients.

Methods: The clinical data of 109 consecutive CRC patients whoreceived robotic surgery at Sun Yat-sen University Cancer Center between June 2016 and May 2019 were retrospectively reviewed. Patient characteristics,tumor traits, treatment details, complications, pathological details, and survival status were evaluated.

Results: Among the 109 patients, 35 (32.1\%) had sigmoid cancer, and 74 (67.9\%) had rectal cancer. Thirty-seven (33.9\%) patients underwent neoadjuvant chemoradiotherapy. Ten (9.2\%) patients underwent sigmoidectomy, 38 (34.9\%) underwent high anterior resection (HAR), 45 (41.3\%) underwent low anterior resection (LAR), and 16 (14.7\%) underwent abdominoperineal resection (APR). The median surgical procedure time was 270 min (range 120 - 465 min). Pathologically complete resection was achieved in all patients. There was no postoperative mortality. Complications occurred in 11 (10.1\%) patients, including $3(2.8 \%)$ anastomotic leakage, $1(0.9 \%)$ anastomotic bleeding, $1(0.9 \%)$ pelvic hemorrhage, $4(3.7 \%)$ intestinal obstruction, 2 (1.8\%) chylous leakage, and 1 (0.9\%) delayed wound union. At a median follow-up of 17 months (range 1-37 months), 1 (0.9\%) patient developed local recurrence and 5 (4.6\%) developed distant metastasis, with one death due to disease progression.

Conclusions: Our results suggest that robotic surgery is technically feasible and safe for Chinese CRC patients, especially for rectal cancer patients who received neoadjuvant treatment. A robotic laparoscope with large magnification showed a clear surgical space for pelvic autonomic nerve preservation in cases of mesorectal edema.

\section{Background}

Colorectal cancer (CRC) is the third most common cancer and a leading cause of cancer death worldwide(Bray et al., 2018), which is an increasingly important obstacle to gains in life expectancy in China(Chen et al., 2016; Feng et al., 2019; Zhang et al., 2017). Despite improvements in the comprehensive treatment and management of CRC patients in recent years, surgery remains the most effective treatment and offers the possibility of a cure for CRC. The quality of surgery is closely associated with oncological outcome. Therefore, a suitable technique for CRC surgery is urgently needed in clinical practice.

Increasing evidence supported by randomized controlled trials demonstrated that laparoscopic surgery was not inferior to open surgery with respect to shortterm surgical outcomes and long-term oncological outcomes(Nelson et al., 2004; Jayne et al., 2007; Guillou et al., 2005; Ng et al., 2008; Lujan et al., 2009), which is becoming the new standard for colorectal cancer treatment. Many advantages of laparoscopic surgery have been reported, including shorter length of stay, smaller scars, and reduced recovery time(Weeks et al., 2002). However, laparoscopic surgery may present some technical drawbacks, such as loss of three-dimensional (3D) view, long instruments that can increase physiological hand tremor, and loss of dexterity. Recently, robot-assisted laparoscopic surgery (RALS) using the Intuitive Surgical ${ }^{\circledR}$ da Vinci ${ }^{\text {Tm }}$ surgical system (Intuitive Surgical ${ }^{\circledR}$, Sunnyvale, CA) was developed to facilitate minimally invasive surgery, and this technique provides a stable 3D view and intuitively transfers movements from the handle to the tip of the instrument with tremor filtering to offer enhanced dexterity(Ballantyne, 2002).

Robotic colorectal surgery has been increasingly performed in recent years, and the safety and feasibility have also been confirmed in previous studies(Pai et al., 2015; Gomez Ruiz et al., 2016; Shiomi et al., 2014). Limited studies have presented clinical data for patients with CRC receiving robotic surgery in China. The aim of this study is to present short-term surgical and oncological outcomes of robotic surgery and further confirm its safety and feasibility in Chinese patients with sigmoid and rectal cancer.

\section{Patients And Methods}

\section{Patient selection}

The medical records of 109 consecutive patients were reviewed. All patients were diagnosed with sigmoid colon or rectal cancer and underwent robotic surgery between June 2016 and May 2019 at Sun Yat-sen University Cancer Center (Guangzhou, China). All cases were staged according to the 8th edition American Joint Committee on Cancer (AJCC) staging system. The following inclusion criteria were used: (1) histologically confirmed sigmoid colon or rectal adenocarcinoma; (2) underwent robotic curative resection of tumor using the da Vinci Surgical System (Intuitive Surgical Inc, Sunnyvale, CA, USA); and (3) had a complete record of the whole treatment. The patient demographics, tumor characteristics, type of procedure performed, comorbid conditions, operative variables, including operative time, conversion to open, lymph nodes retrieved, estimated blood loss, and blood transfusion, and postoperative variables, including length of stay, and 30-day mortality were carefully reviewed, and oncological outcomes were assessed. The present study was performed according to the ethical standards of the World Medical Association Declaration of Helsinki and was approved by the Institutional Review Board and Independent Ethics Committees of Sun Yat-sen University Cancer Center. The informed consent requirement was waived based on the nature of this retrospective study, in which patient data were kept confidential.

\section{Surgical Techniques}

The exact trocar placement is shown in Fig. 1A. There are 4 trocars placed for the surgery: 1 for the camera, 2 for the robotic arms, and 1 for the assistant. A camera port $(12 \mathrm{~mm})$ was placed $3-4 \mathrm{~cm}$ above and to the right of the umbilicus. Robotic arm $1(8 \mathrm{~mm})$ was placed right of the iliac fossa along a line drawn 
from the umbilicus to the anterior superior iliac spine, one third of the way from the anterior superior iliac spine. Robotic arm 2 (8 mm) was placed $3-4 \mathrm{~cm}$ below the xiphoid process. An assistant port was placed $(12 \mathrm{~mm})$ at the intersection of the vertical line through McBurney's point and the horizontal line through the camera port.

Total mesorectal excision (TME) and tumor-specific mesorectal excision (TSME) were performed as previously described(Xu and Qin, 2016). The procedure of pelvic autonomic nerves preservation (PANP) was performed at the same time. The sigmoid mesocolon was cut along the right pararectal sulcus using the middle approach, and the inferior mesenteric artery was fully exposed. The inferior mesenteric artery was clamped and cut off approximately $1 \mathrm{~cm}$ from the root of the blood vessel in order to protect the superior hypogastric plexus. The "cavity effect" of electric heating equipment was quickly exposed, and Toldt's plane was subsequently entered.The white filamentous connective tissue in Toldt's space was cut sharply using an electric knife and kept in the neurosurgical plane of the white filamentous connective tissue at all times. We separated the posterior wall of the rectum closely behind the fascia propria of the rectum under direct vision in order to protect the inferior hypogastric nerve and the anterior sacral vessel. Similarly, sharp separation of the rectal lateral walls was performed near the outer edge of the rectal ligament and the inside edge of the pelvic plexus to protect the pelvic plexus. The anterior rectal space between the anterior and posterior Denonvilliers' fascia was separated to protect the branches of the pelvic plexus.

\section{Follow-up}

Patients were scheduled for subsequent visits every 3 months for 2 years then semiannually until 3 years after surgery. Physical examination, blood tests for carcinoembryonic antigen (CEA) and carbohydrate antigen 19 - 9 (CA19-9) levels, abdominal ultrasonography, and chest X-rays were performed every 3 months postoperatively. Chest/abdominal/pelvic computed tomography (CT) and colonoscopy were performed annually. Disease-free survival (DFS) was defined as the interval from surgery to disease recurrence, death, or the last follow-up.Overall survival (OS) was defined as the interval from the date of surgery until death of any cause or the last follow-up. Patients without any event (metastasis or death) at the last follow-up date were regarded as random censoring. The last follow-up visit was in July 2019.

\section{Statistical analysis}

All statistical analyses were performed using IBM SPSS statistics software, version 21.0 (IBM Corp., Armonk, NY, USA). All of the continuous data are expressed as the means with standard deviation and range. All of the categorical data were calculated as numbers and percentages. The 2-year OS rate and 2year DFS rate were calculated using the Kaplan-Meier method.

\section{Results}

\section{Patient characteristics}

Over a 3-year period, 10 (9.2\%) patients underwent sigmoidectomy, 38 (34.9\%) underwent high anterior resection (HAR), 45 (41.3\%) underwent low anterior resection (LAR), and 16 (14.7\%) underwent abdominoperineal resection (APR) (Fig. 1). Their demographic features and clinicopathological characteristics are summarized in Table 1. Of the total 109 patients, 35 (32.1\%) patients presented with sigmoid colon cancer and 74 (67.9\%) patients had rectal cancer. Seventyfive patients (68.8\%) were males, and 34 (31.2\%) were females, with a median age of 59 years (range, 31-82 years). The mean body mass index (BMI) was $22.8 \pm 3.0$ and comparable between the patients with sigmoid cancer and rectal cancer. Preoperative clinical stage included 17 (15.6\%) stage I, 43 (39.4\%) stage II, 45 (41.3\%) stage III, and 4 (3.7\%) stage IV. Ten (9.2\%) patients underwent sigmoidectomy. Thirty-eight (34.9\%) of the 74 patients with rectal cancer, 37 (50\%) received neoadjuvant chemoradiotherapy (CRT) and 4 (5.4\%) received neoadjuvant chemotherapy. 
Table 1

Clinical characteristics of the study population

\begin{tabular}{|c|c|c|c|}
\hline Variables & $\begin{array}{l}\text { Total } \\
(n=109)\end{array}$ & $\begin{array}{l}\text { Sigmoid cancer } \\
(n=35)\end{array}$ & $\begin{array}{l}\text { Rectal cancer } \\
(n=74)\end{array}$ \\
\hline Age [median (range), years] & $59(31-82)$ & $60(34-82)$ & $57(31-73)$ \\
\hline Age > 65 years $(n, \%)$ & $30(27.5)$ & $10(28.6)$ & $20(27.0)$ \\
\hline Male sex $(n, \%)$ & $75(68.8)$ & $25(71.4)$ & $50(67.6)$ \\
\hline $\mathrm{BMI}\left(\right.$ mean $\left.\pm \mathrm{SD}, \mathrm{kg} / \mathrm{m}^{2}\right)$ & $22.8 \pm 3.0$ & $22.7 \pm 2.8$ & $22.9 \pm 3.2$ \\
\hline$<18.5$ & $5(4.6)$ & $1(2.9)$ & $4(5.4)$ \\
\hline $18.5-23.9$ & $63(57.8)$ & $23(65.7)$ & $40(54.1)$ \\
\hline $24-27.9$ & $36(33.0)$ & $10(28.6)$ & $26(35.1)$ \\
\hline$\geq 28$ & $5(4.6)$ & $1(2.9)$ & $4(5.4)$ \\
\hline \multicolumn{4}{|l|}{ ASA classification $(n, \%)$} \\
\hline 1 & $18(16.5)$ & $5(14.3)$ & $13(17.6)$ \\
\hline 2 & $84(77.1)$ & $26(74.3)$ & $58(78.4)$ \\
\hline 3 & $7(6.4)$ & $4(11.4)$ & $3(4.1)$ \\
\hline Smoking history $(n, \%)$ & $23(21.1)$ & $7(20.0)$ & $16(21.6)$ \\
\hline Hypertension $(n, \%)$ & $29(26.6)$ & $11(31.4)$ & $18(24.3)$ \\
\hline Diabetes mellitus $(n, \%)$ & $14(12.8)$ & $4(11.4)$ & $10(13.5)$ \\
\hline Bowel obstruction $(n, \%)$ & $3(2.8)$ & $2(5.7)$ & $1(1.4)$ \\
\hline Weight loss within 6 months $(n, \%)$ & $27(24.8)$ & $10(28.6)$ & $17(23.0)$ \\
\hline Hemoglobin (mean $\pm \mathrm{SD}, \mathrm{g} / \mathrm{dL}$ ) & $132.2 \pm 17.6$ & $133.2 \pm 20.2$ & $131.7 \pm 16.4$ \\
\hline Severe anemia $(n, \%)$ & $2(1.8)$ & $1(2.9)$ & $1(1.4)$ \\
\hline Albumin (mean $\pm \mathrm{SD}, \mathrm{g} / \mathrm{dL}$ ) & $41.8 \pm 3.6$ & $41.8 \pm 4.2$ & $41.9 \pm 3.4$ \\
\hline Median DAV [median (range), cm] & $10(1-30)$ & $20(16-30)$ & $7(1-15)$ \\
\hline$>15$ & $35(32.1)$ & $35(100)$ & 0 \\
\hline $11-15$ & $13(11.9)$ & 0 & $13(17.6)$ \\
\hline $6-10$ & $35(32.1)$ & 0 & $35(47.3)$ \\
\hline$\leq 5$ & $26(23.9)$ & 0 & $26(35.1)$ \\
\hline \multicolumn{4}{|l|}{ Preoperative TNM stage $(n, \%)$} \\
\hline I & $17(15.6)$ & $4(11.4)$ & $13(17.6)$ \\
\hline ॥ & $43(39.4)$ & $15(42.9)$ & $28(37.8)$ \\
\hline III & $45(41.3)$ & $15(42.9)$ & $30(40.5)$ \\
\hline IV & $4(3.7)$ & $1(2.9)$ & $3(4.1)$ \\
\hline Neoadjuvant CRT $(n, \%)$ & $37(33.9)$ & 0 & $37(50.0)$ \\
\hline Neoadjuvant chemotherapy $(n, \%)$ & $4(3.7)$ & 0 & $4(5.4)$ \\
\hline
\end{tabular}

\section{Intraoperative Outcomes}

The intraoperative outcomes are presented in Table 2. The median operative time for robotic surgery was 270 min, with a range of 120 min to 465 min. Median intraoperative transfusion volume for the total cohort was $2000 \mathrm{ml}$ (range $1000-4500 \mathrm{ml}$ ). Median intraoperative urine volume for the cohort was $400 \mathrm{ml}$ (range 100-2100 ml). Median estimated blood loss for the cohort was $50 \mathrm{ml}$ (range 20-400 ml). Three patients had blood transfusion, including 2 patients in the APR group (12.5\%) and 1 patient in the sigmoidectomy and HAR group (2.1\%). None of the cases was converted to an open or laparoscopic procedure, and no intraoperative ureteral injury occurred. Twenty-two patients underwent preventive ileostomy, including 4 patients in the sigmoidectomy and HAR group (8.3\%) and 18 patients in the LAR group (40.0\%). 
Table 2

Intraoperative outcomes of patients

\begin{tabular}{|c|c|c|c|c|}
\hline Variables & Total $(n=109)$ & Sigmoidectomy + HA $(n=48)$ & $\operatorname{LAR}(n=45)$ & APR $(n=16)$ \\
\hline Procedure time [median (range), minutes] & $270(120-465)$ & $240(120-435)$ & $300(165-450)$ & $295(170-465)$ \\
\hline $\begin{array}{l}\text { Intraoperative transfusion volume [median (range), } \\
\mathrm{mL} \text { ] }\end{array}$ & $\begin{array}{l}2000(1000- \\
4500)\end{array}$ & $2000(1000-3500)$ & $2000(1000-4500)$ & $2500(1500-3300)$ \\
\hline Intraoperative urine volume [median (range), $\mathrm{mL}$ ] & $400(100-2100)$ & $400(100-1600)$ & $350(100-2100)$ & $500(200-2000)$ \\
\hline Estimated blood loss [median (range), mL] & $50(20-400)$ & $50(20-300)$ & $50(50-400)$ & $100(30-300)$ \\
\hline Blood transfusion, $n(\%)$ & $3(2.8)$ & $1(2.1)$ & 0 & $2(12.5)$ \\
\hline Conversion, $n(\%)$ & 0 & 0 & 0 & 0 \\
\hline Ureteral injury, $n(\%)$ & 0 & 0 & 0 & 0 \\
\hline Preventive ileostomy, $n$ (\%) & $22(20.2)$ & $4(8.3)$ & $18(40.0)$ & 0 \\
\hline
\end{tabular}

\section{Pathological Outcomes}

The pathological outcomes are presented in Table 3. Pathological stages were stage 0 in 12 patient, stage I in 27 patients, stage II in 38 patients, stage III in 28 patients and stage IV in 4 patients. There were 41 patients with rectal cancer who had received neoadjuvant treatment, and 11 of these patients exhibited a pathologically complete response ( $\mathrm{pCR}$ ). Pathological complete resection was achieved in all cases. 
Table 3

Pathological outcomes

\begin{tabular}{|c|c|c|c|c|}
\hline \multirow[t]{3}{*}{ Variables } & \multirow{3}{*}{$\begin{array}{l}\text { Total } \\
(n=109)\end{array}$} & \multirow{3}{*}{$\begin{array}{l}\text { Sigmoid colon } \\
\text { cancer } \\
(n=35)\end{array}$} & \multicolumn{2}{|l|}{ Rectal cancer } \\
\hline & & & $\begin{array}{l}\text { Without neoadjuvant } \\
\text { treatment }\end{array}$ & $\begin{array}{l}\text { With neoadjuvant } \\
\text { treatment }\end{array}$ \\
\hline & & & $(n=33)$ & $(n=41)$ \\
\hline Tumor size [median (range), cm] & $2.5(0.5-13.0)$ & $4(10.0-13.0)$ & $2.7(1.5-6.5)$ & $1.5(0.5-5.5)$ \\
\hline \multicolumn{5}{|l|}{ Tumor differentiation, $n(\%)$} \\
\hline No tumor cells & $18(16.5)$ & $4(11.4)$ & $1(3.0)$ & $13(31.7)$ \\
\hline Well-differentiated carcinoma & 0 & 0 & 0 & 0 \\
\hline Moderate carcinoma & $76(69.7)$ & $28(80.0)$ & $25(75.8)$ & $23(56.1)$ \\
\hline Poor carcinoma & $14(12.8)$ & $3(8.6)$ & $7(21.2)$ & $4(9.8)$ \\
\hline Mucous carcinoma & $1(0.9)$ & 0 & 0 & $1(2.4)$ \\
\hline \multicolumn{5}{|l|}{ Pathological T stage, $n(\%)$} \\
\hline Tis & $1(0.9)$ & $1(2.9)$ & 0 & 0 \\
\hline T0 & $12(11.0)$ & 0 & 0 & $12(29.3)$ \\
\hline T1 & $12(11.0)$ & $4(11.4)$ & $8(24.2)$ & 0 \\
\hline T2 & $22(20.2)$ & $2(5.7)$ & $9(27.3)$ & $11(26.8)$ \\
\hline T3 & $52(47.7)$ & $24(68.6)$ & $13(39.4)$ & $15(36.6)$ \\
\hline $\mathrm{T} 4 \mathrm{a}$ & $8(7.3)$ & $3(8.6)$ & $3(9.1)$ & $2(4.9)$ \\
\hline $\mathrm{T} 4 \mathrm{~b}$ & $2(1.8)$ & $1(2.9)$ & 0 & $1(2.4)$ \\
\hline \multicolumn{5}{|l|}{ Pathological N stage, $n(\%)$} \\
\hline NO & $79(72.5)$ & $22(62.9)$ & $21(63.6)$ & $36(87.8)$ \\
\hline $\mathrm{N} 1 \mathrm{a}$ & $13(11.9)$ & $5(14.3)$ & $6(18.2)$ & $2(4.9)$ \\
\hline $\mathrm{N} 1 \mathrm{~b}$ & $5(4.6)$ & $1(2.9)$ & $2(6.1)$ & $2(4.9)$ \\
\hline N1c & $6(5.5)$ & $4(11.4)$ & $1(3.0)$ & $1(2.4)$ \\
\hline $\mathrm{N} 2 \mathrm{a}$ & $4(3.7)$ & $2(5.7)$ & $2(6.1)$ & 0 \\
\hline $\mathrm{N} 2 \mathrm{~b}$ & $2(1.8)$ & $1(2.9)$ & $1(3.0)$ & 0 \\
\hline \multicolumn{5}{|l|}{ Pathological TNM stage, $n(\%)$} \\
\hline 0 & $12(11.0)$ & $1(2.9)$ & 0 & $11(26.8)$ \\
\hline I & $27(24.8)$ & $5(14.3)$ & $12(36.4)$ & $10(24.4)$ \\
\hline II & $38(34.9)$ & $16(45.7)$ & $9(27.3)$ & $13(31.7)$ \\
\hline III & $28(25.7)$ & $12(34.3)$ & $12(36.4)$ & $4(9.8)$ \\
\hline IV & $4(3.7)$ & $1(2.9)$ & 0 & $3(7.3)$ \\
\hline $\begin{array}{l}\text { Positive/total harvested lymph nodes } \\
\text { [median (range), } n]\end{array}$ & $\begin{array}{l}0(0-22) / 12(1- \\
51)\end{array}$ & $\begin{array}{l}0(0-14) / 16(3- \\
33)\end{array}$ & $0(0-22) / 15(5-51)$ & $0(0-2) / 5(1-23)$ \\
\hline $\begin{array}{l}\text { Positive/total central harvested lymph nodes } \\
\text { [median (range), } n \text { ] }\end{array}$ & $0(0-3) / 3(0-26)$ & $0(0-3) / 4(0-12)$ & $0(0) / 4(0-26)$ & $0(0) / 2(0-8)$ \\
\hline $\begin{array}{l}\text { Positive/total intermediate harvested lymph } \\
\text { nodes } \\
\text { [median (range), } n]\end{array}$ & $0(0-3) / 3(0-16)$ & $0(0-3) / 4(0-9)$ & $0(0-2) / 4(0-16)$ & $0(0) / 2(0-9)$ \\
\hline $\begin{array}{l}\text { Positive/total paraintestinal harvested lymph } \\
\text { nodes } \\
\text { [median (range), } n]\end{array}$ & $\begin{array}{l}0(0-22) / 4(0- \\
23)\end{array}$ & $0(0-8) / 7(1-15)$ & $0(0-22) / 6(1-23)$ & $0(0-2) / 2(0-12)$ \\
\hline $\begin{array}{l}\text { Distance of distal resection margin } \\
\text { [median (range), } \mathrm{cm}]\end{array}$ & $5(1.0-10.0)$ & $8(5.0-10.0)$ & $5(1.0-10.0)$ & $2.5(1.0-10.0)$ \\
\hline
\end{tabular}




\begin{tabular}{|c|c|c|c|c|}
\hline \multirow[t]{3}{*}{ Variables } & \multirow{3}{*}{$\begin{array}{l}\text { Total } \\
(n=109)\end{array}$} & \multirow{3}{*}{$\begin{array}{l}\text { Sigmoid colon } \\
\text { cancer } \\
(n=35)\end{array}$} & \multicolumn{2}{|l|}{ Rectal cancer } \\
\hline & & & $\begin{array}{l}\text { Without neoadjuvant } \\
\text { treatment }\end{array}$ & $\begin{array}{l}\text { With neoadjuvant } \\
\text { treatment }\end{array}$ \\
\hline & & & $(n=33)$ & $(n=41)$ \\
\hline Positive resection distal margin, $n$ & 0 & 0 & 0 & 0 \\
\hline
\end{tabular}

\section{Postoperative Outcomes}

As shown in Table 4, the median length of stay (LOS) was 7 (range, 4-30) days. There was no postoperative mortality within 30 days. Eleven (10.1\%) patients suffered complications after surgery, including $3(2.8 \%)$ patients with anastomotic leakage, $1(0.9 \%)$ patient with anastomotic bleeding, 1 (0.9\%) patient with pelvic hemorrhage, 4 (3.7\%) patients with intestinal obstruction, 2 (1.8\%) patients with chylous leakage, and 1 (0.9\%) patient with delayed wound healing. Only 5 (4.6\%) and 8 (7.3\%) patients developed urinary and sexual dysfunction, respectively. Details about the complication events are presented in Table 5. Among the patients who suffered postoperative complications, 2 patients required surgery, and 9 patients received conservative treatment. All of these patients achieved recovery after invention.

Table 4

Postoperative and oncological outcomes

\begin{tabular}{|c|c|c|c|c|}
\hline Variables & $\begin{array}{l}\text { Total } \\
(n=109, \%)\end{array}$ & Sigmoidectomy + HAR $(n=48, \%)$ & $\begin{array}{l}\text { LAR } \\
(n=45 \%)\end{array}$ & $\begin{array}{l}\text { APR } \\
(n=16, \%)\end{array}$ \\
\hline $\begin{array}{l}\text { LOS after surgery } \\
\text { [median (range), days] }\end{array}$ & $7(4-30)$ & $7(4-12)$ & $7(4-24)$ & $8(6-30)$ \\
\hline 30 day mortality & 0 & 0 & 0 & 0 \\
\hline Postoperative complication & $11(10.1)$ & $2(4.2)$ & $7(15.6)$ & $2(12.5)$ \\
\hline Anastomotic leakage & $3(2.8)$ & 0 & $3(6.7)$ & 0 \\
\hline Anastomotic bleeding & $1(0.9)$ & $1(2.1)$ & 0 & 0 \\
\hline Pelvic hemorrhage & $1(0.9)$ & 0 & $1(2.2)$ & 0 \\
\hline Intestinal obstruction & $4(3.7)$ & 0 & $3(6.7)$ & $1(6.3)$ \\
\hline Chylous leakage & $2(1.8)$ & $1(2.1)$ & $1(2.2)$ & 0 \\
\hline Delayed wound healing & $1(0.9)$ & 0 & 0 & $1(6.3)$ \\
\hline Defecation dysfunction & $38(34.9)$ & $9(18.8)$ & $29(64.4)$ & 0 \\
\hline Urinary dysfunction & $5(4.6)$ & 0 & $1(2.2)$ & $4(25.0)$ \\
\hline Sexual dysfunction & $8(7.3)$ & 0 & $4(8.9)$ & $4(25.0)$ \\
\hline Alive (NED) & $102(93.6)$ & 45 (93.8) & $41(91.1)$ & $16(100)$ \\
\hline Alive with tumor & $6(5.5)$ & $2(4.2)$ & $4(8.9)$ & 0 \\
\hline Death due to tumor & $1(0.9)$ & $1(2.1)$ & 0 & 0 \\
\hline Local recurrence & $1(0.9)$ & $1(2.1)$ & 0 & 0 \\
\hline Distant metastasis & $5(4.6)$ & $2(4.2)$ & $3(6.7)$ & 0 \\
\hline
\end{tabular}

Table 5. Summary of postoperative complication events 


\begin{tabular}{|c|c|c|c|c|c|c|c|c|c|c|c|}
\hline Order & Sex & $\begin{array}{l}\text { Age } \\
\text { (years) }\end{array}$ & $\begin{array}{l}\text { Tumor } \\
\text { location }\end{array}$ & $\begin{array}{l}\text { DAV } \\
(\mathrm{cm})\end{array}$ & NACRT & $\begin{array}{l}\text { Pathological } \\
\text { stage }\end{array}$ & $\begin{array}{l}\text { Types of } \\
\text { operation }\end{array}$ & Complication & $\begin{array}{l}\text { Complication } \\
\text { detected on } \\
\text { POD (days) }\end{array}$ & Invention & $\begin{array}{l}\text { Invention } \\
\text { outcome }\end{array}$ \\
\hline 1 & Female & 37 & Rectum & 4 & Yes & T2NOMO & LAR & $\begin{array}{l}\text { Anastomotic } \\
\text { leakage, } \\
\text { Intestinal } \\
\text { obstruction }\end{array}$ & 2 & $\begin{array}{l}\text { Conservative } \\
\text { treatment }\end{array}$ & Recovery \\
\hline 2 & Male & 59 & Rectum & 10 & No & T3N1M0 & LAR & $\begin{array}{l}\text { Pelvic } \\
\text { hemorrhage }\end{array}$ & 5 & $\begin{array}{l}\text { Conservative } \\
\text { treatment }\end{array}$ & Recovery \\
\hline 3 & Male & 47 & Rectum & 6 & Yes & $\mathrm{pCR}$ & LAR & $\begin{array}{l}\text { Intestinal } \\
\text { obstruction }\end{array}$ & 5 & $\begin{array}{l}\text { Conservative } \\
\text { treatment }\end{array}$ & Recovery \\
\hline 4 & Female & 54 & Rectum & 7 & No & T3NOMO & LAR & $\begin{array}{l}\text { Intestinal } \\
\text { obstruction }\end{array}$ & 3 & $\begin{array}{l}\text { Conservative } \\
\text { treatment }\end{array}$ & Recovery \\
\hline 5 & Female & 37 & $\begin{array}{l}\text { Sigmoid } \\
\text { colon }\end{array}$ & 25 & No & T3NOMO & HAR & $\begin{array}{l}\text { Chylous } \\
\text { leakage }\end{array}$ & 3 & $\begin{array}{l}\text { Conservative } \\
\text { treatment }\end{array}$ & Recovery \\
\hline 6 & Male & 69 & Rectum & 5 & Yes & T3NOMO & LAR & $\begin{array}{l}\text { Chylous } \\
\text { leakage }\end{array}$ & 6 & $\begin{array}{l}\text { Conservative } \\
\text { treatment }\end{array}$ & Recovery \\
\hline 7 & Male & 43 & Rectum & 3 & Yes & $\mathrm{pCR}$ & APR & $\begin{array}{l}\text { Intestinal } \\
\text { obstruction }\end{array}$ & 3 & Operation & Recovery \\
\hline 8 & Male & 50 & Rectum & 1 & Yes & T4N0MO & APR & $\begin{array}{l}\text { Delay wound } \\
\text { healing }\end{array}$ & 8 & $\begin{array}{l}\text { Conservative } \\
\text { treatment }\end{array}$ & Recovery \\
\hline 9 & Male & 68 & $\begin{array}{l}\text { Sigmoid } \\
\text { colon }\end{array}$ & 28 & No & T3N2M0 & Sigmoidectomy & $\begin{array}{l}\text { Anastomotic } \\
\text { bleeding }\end{array}$ & 1 & $\begin{array}{l}\text { Conservative } \\
\text { treatment }\end{array}$ & Recovery \\
\hline 10 & Male & 49 & Rectum & 10 & No & T3N1M0 & LAR & $\begin{array}{l}\text { Anastomotic } \\
\text { leakage }\end{array}$ & 6 & $\begin{array}{l}\text { Conservative } \\
\text { treatment }\end{array}$ & Recovery \\
\hline 11 & Female & 59 & Rectum & 6 & Yes & T3N0M1 & LAR & $\begin{array}{l}\text { Anastomotic } \\
\text { leakage }\end{array}$ & 5 & Operation & Recovery \\
\hline
\end{tabular}

Abbreviations: DAV, inferior tumor margin from the anal verge; NACRT, neoadjuvant chemoradiotherapy; POD, postoperative day; LOS, length of stay; pCR, pathological complete response; HAR, high anterior resection; LAR, low anterior resection; APR, abdominoperineal resection; NED, no evidence of disease.

\section{Survival Analysis}

The median follow-up period for all patients was 17 months (range 1-37 months). One hundred and two patients (93.6\%) in our study cohort were alive with no evidence of disease. One (0.9\%) patient developed local recurrence, and 5 (4.6) patients developed distant metastasis. One patient died due to disease progression. The 2-year OS rate of all patients ( $n=109$ ) was $97.2 \%$ (Fig. 3A), and the 2-year DFS rate of nonmetastatic patients ( $n=104)$ was $92.9 \%$ (Figure. 3B). The 2-year DFS rate of patients in stages 0 , I, II, and III were $100 \%, 95.5 \%, 90.5 \%$, and $88.8 \%$, respectively (Figure. 3C).

\section{Discussion}

In this retrospective study, we investigated the surgical and oncological outcomes of robotic resection for sigmoid and rectal cancer in Chinese patients. Our data found that robotic surgery had a low conversion rate, low morbidity rate, and remarkable oncological outcomes, which confirms its safety and feasibility in Chinese patients with sigmoid and rectal cancer.

Rectal cancer resection is very difficult to perform using traditional laparotomy, but laparoscopic surgery has an advantage for rectal surgery under a clearer view despite the narrow and deep pelvic space. Several studies(Pai et al., 2015; Gomez Ruiz et al., 2016; Shiomi et al., 2014; Tang et al., 2017; Park et al., 2011) confirmed that laparoscopic surgery presented better short-term outcomes and comparable long-term outcomes compared to traditional laparotomy. The surgical advantages and comparable oncological outcomes of laparoscopic surgery were clearly demonstrated in patients with locally advanced rectal cancer after preoperative chemoradiotherapy in the COREAN trial(Jeong et al., 2014). Because of the features of robotic technology, robotic surgery is much more advantageous, especially for patients with locally advanced rectal cancer after treatment with preoperative chemoradiotherapy. In our study, $37.6 \%$ patients presented with a BMI $\geq 24 \mathrm{~kg} / \mathrm{m}^{2}$, and $55.4 \%$ patients with rectal cancer received neoadjuvant treatment. No conversion occurred with a median procedure time of $270 \mathrm{~min}$, a median estimated blood loss of $50 \mathrm{ml}$ and a median length of stay of 7 days. Only 11 patients (10.1\%) experienced postoperative complications, which shows the remarkable surgical advantages of robotic surgery in patients with rectal cancer who received neoadjuvant treatment.

As previously reported, the most commonly encountered complication was anastomotic leakage, and its average occurrence rate was $8.6 \%$ (range from 1.220.5\%)(Trastulli et al., 2012; Cong et al., 2013) and 1.8 to 13.6\% in robotic surgery(Kwak et al., 2011; Baik et al., 2009). Its occurrence affects the patient's quality of life, increases hospitalization costs, delays the implementation of adjuvant chemotherapy, and shortens the overall survival(Baik et al., 2009; Kulu et al., 2015). Eleven patients (10.1\%) had postoperative complications, which included 3 patients who suffered anastomotic leakage. Due to the advantages of 
robotic surgery, such as 3D magnified view, wristed instruments and stable camera platform, surgeons are able to maintain the sufficient surgical dissection plane down to the pelvic floor, which minimizes damage to marginal vessels and allows performance of the rectal division and reconstruction efficiently and safely to shorten the procedure time.

More precise surgery also helps protect the autonomic nerves and reduce the occurrence of long-term postoperative complications, including defecation, urinary and sexual dysfunction(Shiomi et al., 2014). Jiang and coworkers(Wang et al., 2017) described a significant increase in International Prostate Symptom Score (IPSS) after surgery in the laparoscopic group, and more patients in the laparoscopic group (34.8\%) perceived a severe damage in their overall level of sexual function following surgery than the patients in the robotic group (18.3\%). Several studies(Kim et al., 2012; Luca et al., 2013) also claimed that robotic TME improved the preservation of urinary and sexual functions because the arms of the robotic device are stable and highly flexible in the separation and exposure of tissues. With the high-resolution lens of the da Vinci surgical system to effectively recognize the nerve, the application of the PANP technique resulted in a significant reduction in the incidence of urinary dysfunction (4.6\%) and sexual dysfunction (7.3\%) in our study.

A positive circumferential margin or insufficient harvested lymph nodes leads to local recurrence(Marinatou et al., 2014). Although the relationship between sufficiently harvested lymph nodes and local recurrence rate is controversial, the guidelines list the harvesting of $<12$ lymph nodes as risk factor and noted that the performance of TME with clear surgical margins and adequate lymph node dissection were related to lower recurrence rate(Benson et al., 2018a; Benson et al., 2018b). In our study, the median positive total harvested lymph nodes was 0 (range 0-22), and the total harvested lymph nodes was 12 (range 1 -51). The 2 -year DFS of patients in stages $0, \mathrm{I}, \mathrm{II}$, and III were $100 \%, 95.5 \%, 90.5 \%$, and $88.8 \%$, respectively, and the 2 -year DFS of patients in stage III was slightly better than previous studies (65.2\% - 82.8\%)(Pai et al., 2015; Gomez Ruiz et al., 2016; Park et al., 2011). The high quality of the procedure (no positive resection distal margin and sufficient harvested lymph nodes) and neoadjuvant treatment contributed to the remarkable oncological outcomes.

Several limitations should be acknowledged in the present study. First, this retrospective descriptive study included an uncontrolled, single-arm methodology and a limited number of patients from a single cohort. Although our study confirms the safety and feasibility of robotic surgery in Chinese CRC patients, the findings must be validated in a prospective, multicenter clinical trial with a large population in the future. Second, the short follow-up duration was insufficient to evaluate 5-year survival outcomes, which may have led to a misestimation of the effect of robotic surgery on OS and DFS. Additionally, selective bias undeniably exists in our cohort.

\section{Conclusion}

Robotic surgery is technically feasible and safe for Chinese CRC patients, especially for rectal cancer patients receiving neoadjuvant treatment because a robotic laparoscope with large magnification shows a clear surgical space for tumor resection in cases of mesorectal edema. Due to the advantages of robotic surgery, surgeons are able to perform the procedure efficiently and safely and help protect marginal vessels and the autonomic nerves, which reduces the occurrence of short-term and long-term postoperative complications and ensures clear surgical margins and adequate lymph node dissection.

\section{Declarations}

\section{Ethics approval and consent to participate}

The present study was performed according to the ethical standards of the World Medical Association Declaration of Helsinki and was approved by the Institutional Review Board and Independent Ethics Committees of Sun Yat-sen University Cancer Center. The informed consent requirement was waived by the ethics committees based on the nature of this retrospective study, in which patient data were kept confidential.

\section{Availability of data and material}

The datasets used and analyzed during the current study are available from the corresponding author on reasonable request. The authenticity of this article has been validated by uploading the key raw data onto the Research Data Deposit public platform (www. researchdata.org.cn).

\section{Competing interests}

The authors declare that they have no competing interests.

\section{Funding}

This work was funded by grants from the National Natural Science Foundation of China (grant No. 81772595), the Science and Technology Planning Project of Guangdong Province (grant No. 2013B090800047) and Guangzhou Science and Technology Plan Projects (Health Medical Collaborative Innovation Program of Guangzhou) (grant No. 201803040019).

\section{Authors' contributions}

Jianhong Peng and Weihao Li analyzed and interpreted the data. Junzhong Lin and Zhizhong Pan were the chief surgeons who performed the surgery and the chemotherapy, and all authors participated. Jianhong Peng, Weihao Li, Junzhong Lin, and Zhizhong Pan were major contributors in writing the manuscript.Jianhong Peng, Weihao Li, Jinghua Tang, Yuan Li, Xueying Li, Xiaojun Wu, Zhenhai Lu, Junzhong Lin, Zhizhong Pan read and approved the final manuscript.

\section{Acknowledgments}


We deeply appreciate the help from all of our colleagues of the Department of Colorectal Surgery at Sun Yat-sen University Cancer Center who were involved in performing the treatments for the current study. Part of the results and abstract of presenting research have been published as meeting conference papers in the $11^{\text {th }}$ Worldwide Congress of Clinical Robotic Surgery Association and the EUROPEAN COLORECTAL CONGRESS 2019.

\section{References}

Baik SH, Kwon HY, Kim JS, et al. (2009) Robotic versus laparoscopic low anterior resection of rectal cancer: short-term outcome of a prospective comparative study. Ann Surg Oncol 16: 1480-1487.

Ballantyne GH. (2002) Robotic surgery, telerobotic surgery, telepresence, and telementoring. Review of early clinical results. Surg Endosc 16: $1389-1402$.

Benson AB, Venook AP, Al-Hawary MM, et al. (2018a) NCCN Guidelines Insights: Colon Cancer, Version 2.2018. J Nat/ Compr Canc Netw 16: $359-369$.

Benson AB, Venook AP, Al-Hawary MM, et al. (2018b) Rectal Cancer, Version 2.2018, NCCN Clinical Practice Guidelines in Oncology. J Natl Compr Canc Netw 16: 874-901.

Bray F, Ferlay J, Soerjomataram I, et al. (2018) Global cancer statistics 2018: GLOBOCAN estimates of incidence and mortality worldwide for 36 cancers in 185 countries. CA Cancer J Clin 68: 394-424.

Chen W, Zheng R, Baade PD, et al. (2016) Cancer statistics in China, 2015. CA Cancer J Clin 66: 115-132.

Cong ZJ, Hu LH, Bian ZQ, et al. (2013) Systematic review of anastomotic leakage rate according to an international grading system following anterior resection for rectal cancer. PLoS One 8: e75519.

Feng RM, Zong YN, Cao SM, et al. (2019) Current cancer situation in China: good or bad news from the 2018 Global Cancer Statistics? Cancer Commun (Lond) 39: 22.

Gomez Ruiz M, Alonso Martin J, Cagigas Fernandez C, et al. (2016) Short- and mid-term outcomes of robotic-assisted total mesorectal excision for the treatment of rectal cancer. Our experience after 198 consecutive cases. Eur J Surg Oncol 42: 848-854.

Guillou PJ, Quirke P, Thorpe H, et al. (2005) Short-term endpoints of conventional versus laparoscopic-assisted surgery in patients with colorectal cancer (MRC CLASICC trial): multicentre, randomised controlled trial. Lancet 365: 1718-1726.

Jayne DG, Guillou PJ, Thorpe H, et al. (2007) Randomized trial of laparoscopic-assisted resection of colorectal carcinoma: 3-year results of the UK MRC CLASICC Trial Group. J Clin Oncol 25: 3061-3068.

Jeong SY, Park JW, Nam BH, et al. (2014) Open versus laparoscopic surgery for mid-rectal or low-rectal cancer after neoadjuvant chemoradiotherapy (COREAN trial): survival outcomes of an open-label, non-inferiority, randomised controlled trial. Lancet Oncol 15: 767-774.

Kim JY, Kim NK, Lee KY, et al. (2012) A comparative study of voiding and sexual function after total mesorectal excision with autonomic nerve preservation for rectal cancer: laparoscopic versus robotic surgery. Ann Surg Oncol 19: 2485-2493.

Kulu Y, Tarantio I, Warschkow R, et al. (2015) Anastomotic leakage is associated with impaired overall and disease-free survival after curative rectal cancer resection: a propensity score analysis. Ann Surg Oncol 22: 2059-2067.

Kwak JM, Kim SH, Kim J, et al. (2011) Robotic vs laparoscopic resection of rectal cancer: short-term outcomes of a case-control study. Dis Colon Rectum 54: $151-156$.

Luca F, Valvo M, Ghezzi TL, et al. (2013) Impact of robotic surgery on sexual and urinary functions after fully robotic nerve-sparing total mesorectal excision for rectal cancer. Ann Surg 257: 672-678.

Lujan J, Valero G, Hernandez Q, et al. (2009) Randomized clinical trial comparing laparoscopic and open surgery in patients with rectal cancer. Br J Surg 96: 982-989.

Marinatou A, Theodoropoulos GE, Karanika S, et al. (2014) Do anastomotic leaks impair postoperative health-related quality of life after rectal cancer surgery? A case-matched study. Dis Colon Rectum 57: 158-166.

Nelson H, Sargent DJ, Wieand HS, et al. (2004) A comparison of laparoscopically assisted and open colectomy for colon cancer. N Engl J Med 350: 20502059.

$\mathrm{Ng}$ SS, Leung KL, Lee JF, et al. (2008) Laparoscopic-assisted versus open abdominoperineal resection for low rectal cancer: a prospective randomized trial. Ann Surg Oncol 15: 2418-2425.

Pai A, Marecik SJ, Park JJ, et al. (2015) Oncologic and Clinicopathologic Outcomes of Robot-Assisted Total Mesorectal Excision for Rectal Cancer. Dis Colon Rectum 58: 659-667. 
Park JS, Choi GS, Lim KH, et al. (2011) S052: a comparison of robot-assisted, laparoscopic, and open surgery in the treatment of rectal cancer. Surg EndosC 25: 240-248.

Shiomi A, Kinugasa Y, Yamaguchi T, et al. (2014) Robot-assisted rectal cancer surgery: short-term outcomes for 113 consecutive patients. Int J Colorectal Dis 29: 1105-1111.

Tang B, Zhang C, Li C, et al. (2017) Robotic Total Mesorectal Excision for Rectal Cancer: A Series of 392 Cases and Mid-Term Outcomes from A Single Center in China. J Gastrointest Surg 21: 569-576.

Trastulli S, Farinella E, Cirocchi R, et al. (2012) Robotic resection compared with laparoscopic rectal resection for cancer: systematic review and meta-analysis of short-term outcome. Colorectal Dis 14: e134-156.

Wang G, Wang Z, Jiang Z, et al. (2017) Male urinary and sexual function after robotic pelvic autonomic nerve-preserving surgery for rectal cancer. Int J Med Robot 13 .

Weeks JC, Nelson H, Gelber S, et al. (2002) Short-term quality-of-life outcomes following laparoscopic-assisted colectomy vs open colectomy for colon cancer: a randomized trial. Jama 287: 321-328.

Xu J and Qin X. (2016) Expert consensus on robotic surgery for colorectal cancer (2015 edition). Chin J Cancer 35: 23.

Zhang Y, Chen Z and Li J. (2017) The current status of treatment for colorectal cancer in China: A systematic review. Medicine (Baltimore) 96 : e8242.

\section{Figures}

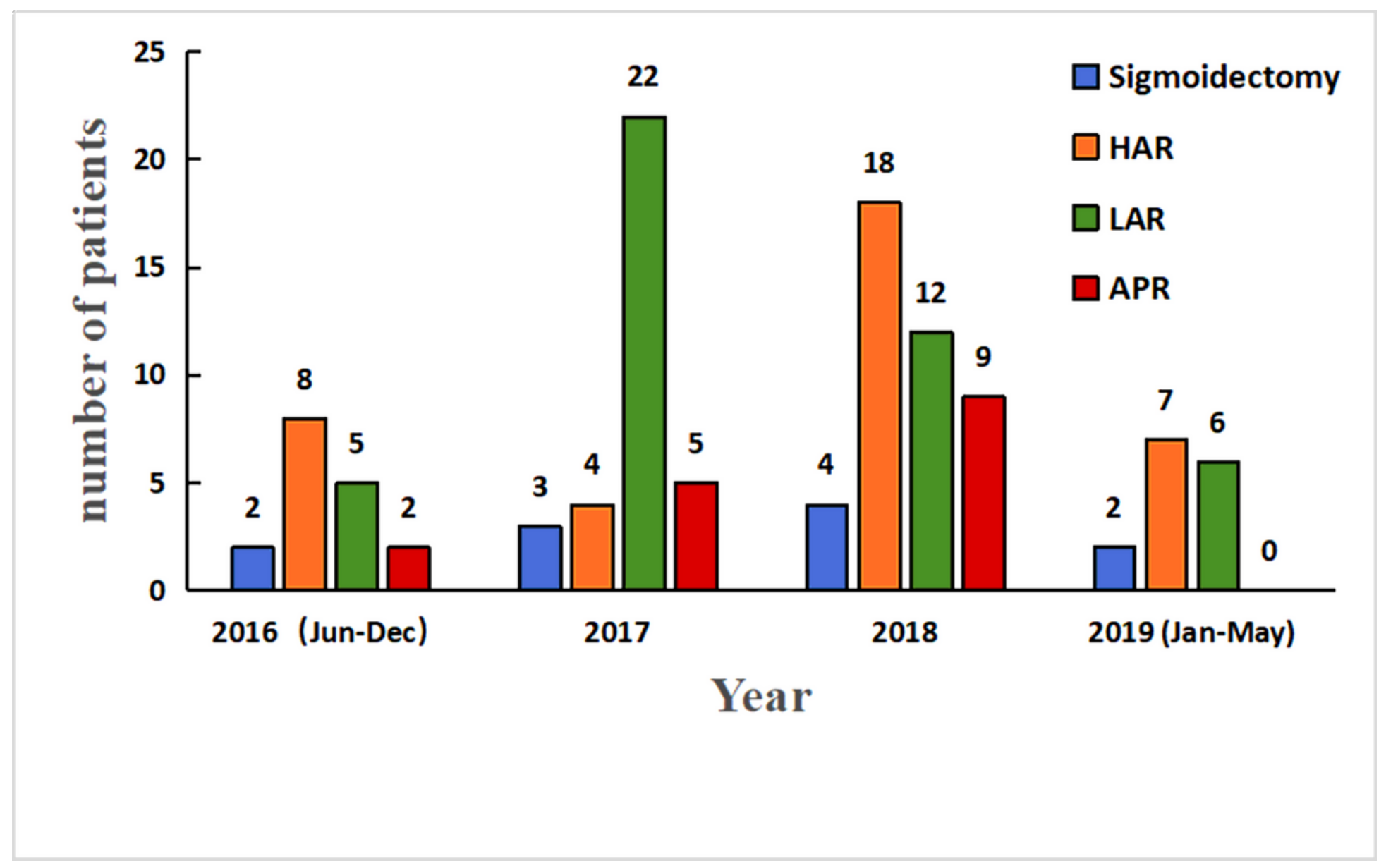

Figure 1

Histogram depicting year-wise distribution of robotic sigmoidectomy, HAR (high anterior resection), LAR (low anterior resection) and APR (abdominoperineal resection) for colorectal cancer. 


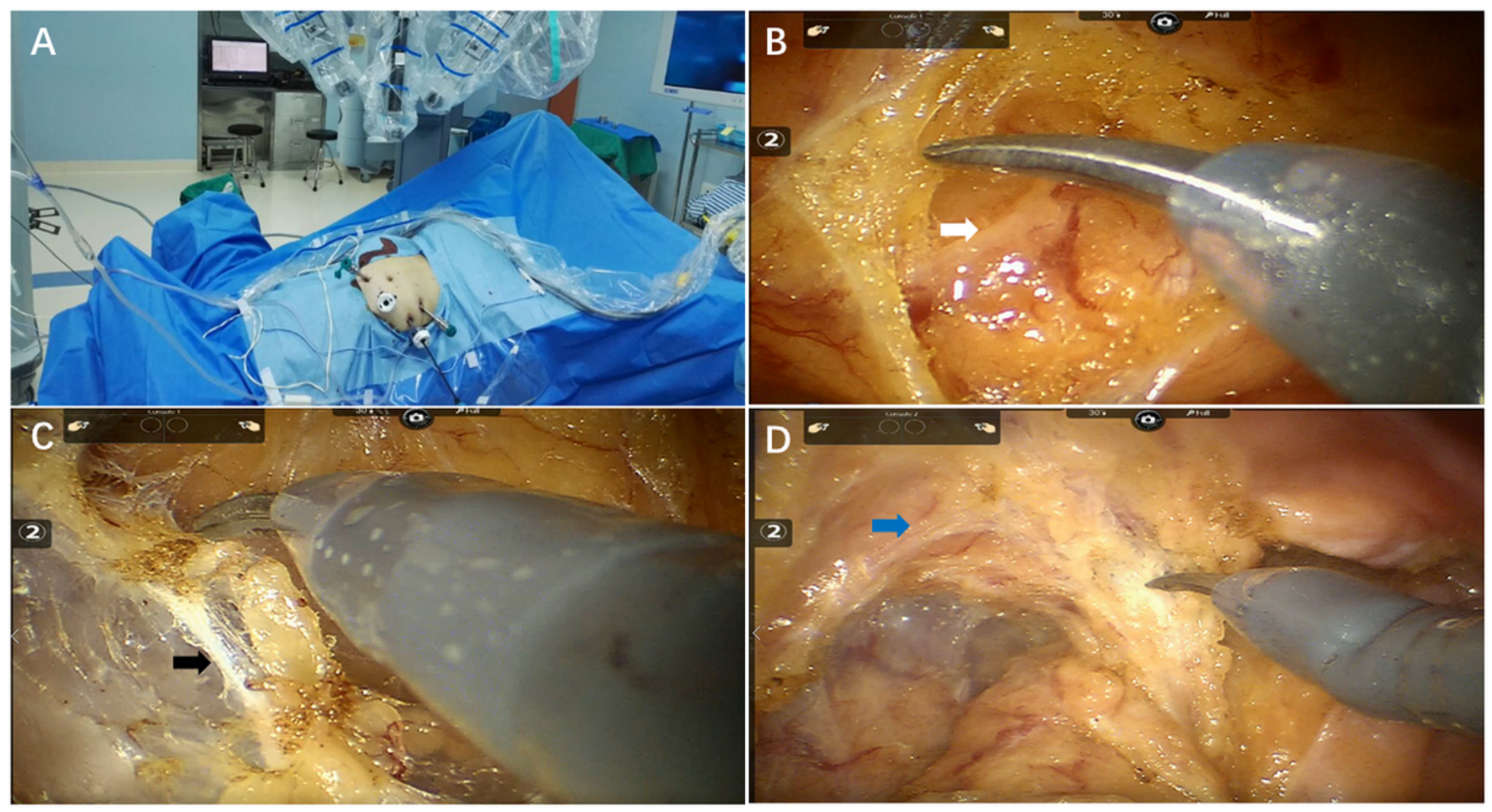

\section{Figure 2}

Key techniques of total mesorectal excision after chemoradiotherapy for pelvic autonomic nerve preservation. (A)Operation room setup (B) Inferior mesenteric nerve (white arrow) preservation(C) Hypogastric nerves (black arrow) preservation (D) Pelvic plexus (blue arrow) preservation. 
A

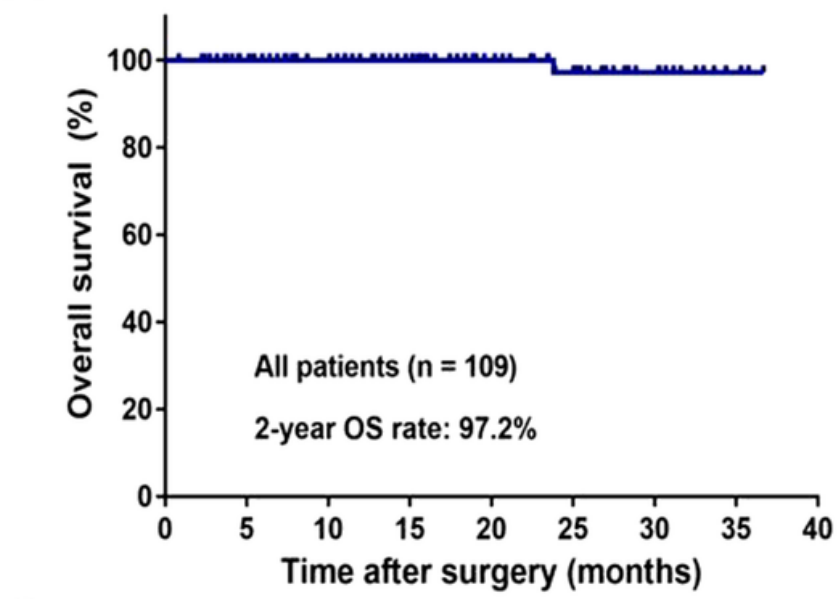

C

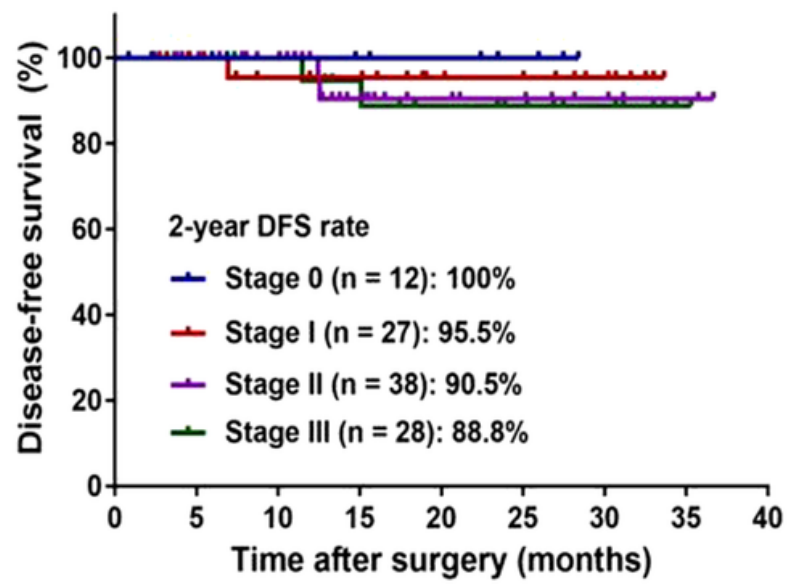

B

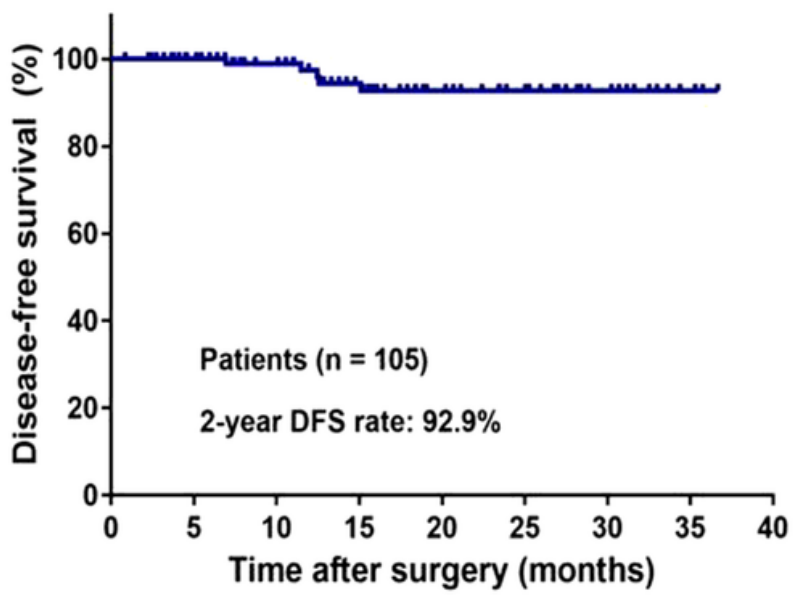

Figure 3

Kaplan-Meier curves of the patients with colorectal cancer underwent robotic surgery. (A) 2-year overall survival for whole study population. (B) 2-year diseasefree survival for non-metastatic patients. (C) 2-year disease-free survival by pathologic stage. 